\title{
Exoplanet telescope diffracted light minimized: the pinwheel-pupil solution
}

James B. Breckinridge, James E. Harvey, Karlton Crabtree, Tony Hull

James B. Breckinridge, James E. Harvey, Karlton Crabtree, Tony Hull, "Exoplanet telescope diffracted light minimized: the pinwheel-pupil solution," Proc. SPIE 10698, Space Telescopes and Instrumentation 2018: Optical, Infrared, and Millimeter Wave, 106981P (6 July 2018); doi: 10.1117/12.2311811

Event: SPIE Astronomical Telescopes + Instrumentation, 2018, Austin, Texas, United States 


\title{
ExoPlanet telescope diffracted light minimized: The pinwheel-pupil solution
}

\author{
James B. Breckinridge ${ }^{1,2}$, James E. Harvey ${ }^{3}$, Karlton Crabtree $^{3}$ and Tony Hull ${ }^{4}$ \\ 1. Breckinridge Associates, LLC, 2. Graduate aeronautical laboratory, Caltech. 3 Photon engineering, LLC, 310 \\ S Williams Blvd \#222. Tucson, AZ 857114. 4. University of New Mexico, Albuquerque, NM.
}

\begin{abstract}
Terrestrial exoplanets shine in light reflected from a parent star. Optical spectra are required to provide evidence of a life-supporting environment. Exoplanets are very faint and their optical spectra are contaminated by the spectrum of the parent star. High angular resolution provided by large apertures is needed to distinguish between the spectrum of the exoplanet and its star. Today, large aperture telescopes use segmented primary mirrors that employ close-packed hexagonal segments. The telescope primary mirror is periodically discontinuous with straight lines. These discontinuities scatter unwanted radiation from the much brighter parent star across the field of view to obscure the light from the very faint terrestrial exoplanet. These discontinuities, which mimic a diffraction grating, result in a non-uniform distribution of background light across the image plane. This non-uniformity masks or hides exoplanets from view, to reduce the number of exoplanets that can be observed with a large aperture telescope or to reduce the quality of spectra and thus lead to misinterpretation of data.

Here we introduce the concept of the pinwheel pupil whose unique diffraction pattern significantly reduces the non-uniform distribution of background radiation. Diffraction patterns from pinwheel pupils are compared to the monolithic filled aperture, the classical Cassegrain, the 60-degree symmetry of the hexagonal segments (JWST, E-ELT, etc.). Diffraction "spikes" are reduced by at least $10^{5}$. We discuss the "pinwheel pupil" advantages to spectroscopy, image processing, and observatory operations. We show that, segment fabrication of curved-sided mirrors is not more difficult than fabrication of hexagonal mirror segments. .

This is the report of quantitative study of Fraunhofer (far field) diffraction patterns produced by three different topologies or architectures of mirror segmentation, when illuminated by a plane wave of monochromatic white-light. A plot, in angular units of the intensity as a function of azimuth, Phi , within annular rings at different FOVs, centered on the system axis of the diffraction pattern will be presented. The advantages of the segmented pinwheel pupil is discussed.
\end{abstract}

Keywords: Telescopes, Exoplanet imaging, segmented mirrors, pinwheel pupil, diffraction control, scattered light, starlight suppression, structures

\subsection{INTRODUCTION}

Direct imaging of terrestrial exoplanets is necessary if astronomers are to obtain detailed spectra of a planet's surface and atmosphere to characterize planetary evolutionary tracks and estimate the probability that the planet is capable of supporting life. The apparent angular separation between the exoplanet and its parent star is less than one-arc second. Exoplanets are much smaller in size than the parent star and shine in light reflected from that star. The ratio of light from the planet to that of the star is between $10^{-10}$ and $10^{-11}$, depending on the size of the planet and its separation.

Space telescope apertures of 15 to 30 meters are needed to provide the angular resolution and the radiationgathering power to produce a significant statistical sample of terrestrial exoplanets for analysis. A telescope aperture of this size cannot be placed in orbit fully erected, rather mirror segments are either folded into a smaller volume (as was done for JWST) or in the future may be assembled in space ${ }^{1}$. Therefore, space telescopes of 15 to 30-meter aperture will be partitioned into individual segments. To allow for deployment or assembly in space, the telescope aperture is divided up into separate mirrors with gaps between mirrors to produce a discontinu ous concave surface. These gaps diffract light across the image plane. If these gaps form periodic linear structures across the aperture, then this diffracted light produces structured, unwanted radiation across the image to mask exoplanets.

The standard space telescope architecture today is that of a Cassegrain telescope, which has a secondary mirror supported by 4 or 3 structural beams that shadow the telescope aperture, as well as a hole on the primary mirror to allow light reflected from the secondary to pass thought and into a science instrument module. The NASA next generation large telescope architecture: Large UV Optical IR (LUVOIR) uses this Cassegrain telescope

Space Telescopes and Instrumentation 2018: Optical, Infrared, and Millimeter Wave, edited by Makenzie Lystrup,

Howard A. MacEwen, Giovanni G. Fazio, Proc. of SPIE Vol. 10698, 106981P

(C) 2018 SPIE · CCC code: $0277-786 X / 18 / \$ 18 \cdot$ doi: $10.1117 / 12.2311811$ 
architecture, along with a primary mirror that is divided into regular hexagonal shaped segments. In this case, the telescope entrance pupil is discontinuous because of both the segment gaps and the secondary support structure shadows.

In this paper, combined with the following paper ${ }^{2}$ we show that by using curved structures for the secondary support system and curved sides to nest the segments one adjacent to the other across the primary mirror, we nearly eliminate the image plane "diffraction-noise" by disrupting the diffraction pattern and creating a nearly uniform background across the image plane.

The advantages of implementing this architecture are: 1. Eliminate the need for exotic and absorbing apodizing masks which control diffraction from segment gaps, 2. Increase exoplanet characterization data quality, 3. Improve radiometric calibration, 4. More accurate image restoration, since the point spread function (PSF) is both rotationally symmetric and isoplanatic over a FOV.

This paper is organized into the following sections:

\section{Introduction}

2. Where are the terrestrial exoplanets? This section shows where within the telescope FOV we will find earth twins;

3. Physical optics of diffraction.; This section presents telescope diffraction patterns from two common telescope architectures (HST and a TMT/ELT-like system).

4. Curved secondary support structures not new. Review of historical literature showing the advantages of curved gaps ond shadows.

5. Candidate aperture. Presents a candidate large telescope aperture architecture and shows preliminary calculation of the scattered light within an annular ring at 0.75 -arc seconds

6. Mirror segment manufacture. Material is presented to show that mirror segments nested with curved sides may be no more difficult to fabricate than those with straight sides that are used in hexagon segmented primary mirrors.

\section{WHERE ARE THE TERRESTRIAL EXOPLANETS?}

To identify terrestrial exoplanet in the FOV we calculate the FOV location of an Earth twin at the distance of the parent star. We estimate its's detectability by calculating the telescope aperture in meters needed to place the Earth twin at the first and third ring of the Airy diffraction pattern at the image plane. The contents of table 1 identifies the location of terrestrial exoplanets and shows telescope apertures needed to record them. Note we assume the exoplanet is at elongation in its orbit, a position the planet occupies only a short time. This makes it very important that we know the orbital details of exoplanet systems we observe to maximize our probability of getting good spectra. 
Table 1 Annular FOV location of Earth-twin terrestrial-exoplanets at elongation as a function of distance in parsecs is shown. Column 1 gives the distance from our solar system to a candidate exoplanet system. Column 2 shows the apparent angular separation between the primary star and its terrestrial exoplanet at elongation in its orbit. Column 3 presents the telescope aperture needed to just resolve this separation. Column 4 gives the telescope aperture needed to place the exoplanet at the third diffraction ring. In both cases the telescope apertures are calculated for 500-nm wavelength.

\begin{tabular}{|c|c|c|c|}
\hline $\begin{array}{c}\text { Distance } \\
\text { Parsecs } \\
\text { PC }\end{array}$ & $\begin{array}{c}\text { Angle } \\
\text { between star } \\
\text { \& Earth twin } \\
\text { in milli-arc - } \\
\text { sec }\end{array}$ & $\begin{array}{c}\text { Aperture in } \\
\text { meters } \\
\text { Diffraction } \\
\text { limited at 500nm }\end{array}$ & $\begin{array}{c}\text { Aperture in } \\
\text { meters third } \\
\text { Airy diffraction } \\
\text { ring }\end{array}$ \\
\hline 10 & 100.0 & 1.2 & 3.7 \\
\hline 20 & 50.0 & 2.5 & 7.5 \\
\hline 30 & 33.3 & 3.7 & 11.1 \\
\hline 40 & 25.0 & 5.0 & 15.0 \\
\hline 50 & 20.0 & 6.2 & 18.6 \\
\hline 60 & 16.7 & 7.4 & 22.2 \\
\hline 70 & 14.3 & 8.7 & 26.1 \\
\hline 80 & 12.5 & 9.9 & 29.7 \\
\hline 90 & 11.1 & 11.1 & 33.3 \\
\hline 100 & 10.0 & 12.0 & 36.0 \\
\hline
\end{tabular}

The Hipparcos catalog shows that there are 2347 stars with measured parallaxes of pi $=33.33$ mas, which correspond to a distance of $30 \mathrm{pc}$ down to stellar magnitude $\mathrm{V}=8^{3}$. Exoplanets are $\sim 10^{10}$ fainter than their parent star. If the parent star has magnitude $V=8$, then the faintest terrestrial exoplanets within $30 \mathrm{pc}$ will be between stellar magnitudes 31 and 34. For reference, the Hubble (2.4-m) ultra-deep field magnitude limit is $\sim 29$ and required an exposure of approximately $2 * 10^{+5}$ seconds $^{4}$.

\section{PHYSICAL OPTICS OF DIFFRACTION}

\section{Ground and space telescopes}

Discontinuous telescope pupils, that is telescope apertures that have some portion of the aperture blocked are responsible for diffraction "noise" at the image plane. Breckinridge, Kuper and Shack (1982) ${ }^{5}$ were the first to discuss the role of secondary support diffraction spikes in finding exoplanets. Figure 2 below shows the diffraction pattern from the HST caused by the secondary support structure. Near the star we also see the "diffuse-light" effects of narrow angle scattered light. The sources of narrow angle scattered light in telescope/coronagraph systems were discussed by Harvey, et. al. ${ }^{6}$

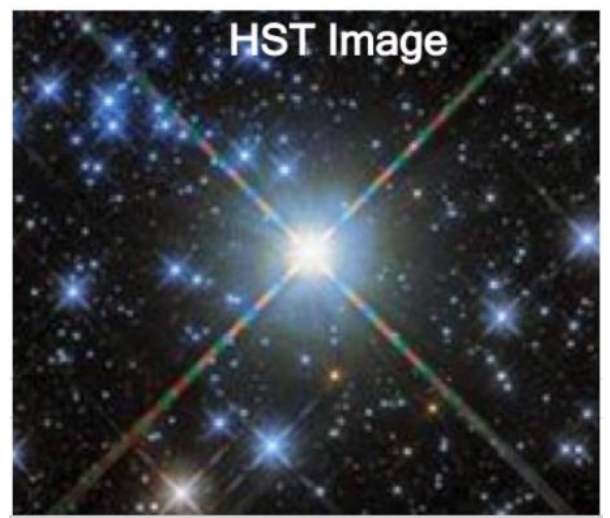

Figure 2 HST image of a star showing the diffraction spikes that mask exoplanets at 4 position angles. The halo around the star is produced in the telescope/instrument system by narrow angle scattered light. The Airy diffraction pattern for HST is about 100 milliarc seconds which is too large to observe terrestrial exoplanets. 
Figure 3 shows the pupil, left and the irradiance at the image plane for an on-axis star at 1-micron wavelength for the 30-meter diameter CELT (now called the Thirty Meter Telescope, TMT) 7 . The PSF is plotted on a $\log _{10}$ intensity scale and the grey scale across the top of the PSF image on the right shows intensity order of magnitude from $10^{0}$ to $10^{-10}$. The field of view is $1 \times 1$ arc-second. We see light scattered beyond 1 arc-second at intensities greater than $10^{-4}$ to obscure accurate exoplanet radiometric and spectral measurements. This scattered light is caused by the periodic structure across the telescope primary mirror produced by the close-packet hexagonal segments. Clearly if we can devise a pupil architecture or topology to mitigate this prominent diffraction pattern science data quality and exoplanet yield will increase.

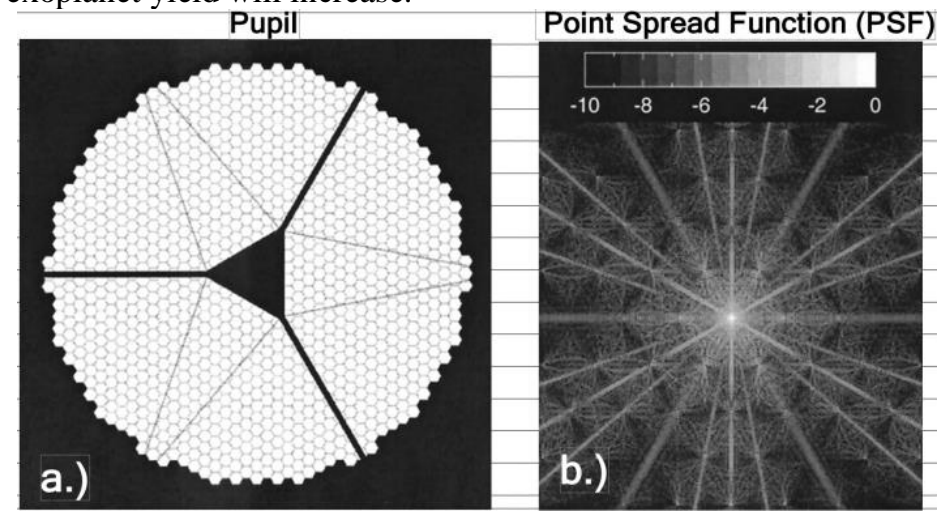

Figure 3. a.) Illustration of the proposed $30 \mathrm{~m}$ diameter pupil of the CELT (now called the Thirtymeter telescope), complete with segmentation geometry, central obscuration and associated secondary mirror struts; b.) Monochromatic logarithmic PSF (wavelength=1 micron) for this Image. The field of view is $1 \times 1$ arcsecond. Diffraction effects of the triangular central obscuration and the secondary mirror struts are readily apparent, as are the characteristic hexagonal symmetry of the mirror perimeter and the inter-segment gaps.

\section{Segmented space telescope diffraction}

Space telescopes that are segmented use close-packed regular hexagon-sided mirrors to pack into a nearly circular telescope pupil. Figure 4 below shows, on the left, one of the concept apertures for the Large UltraViolet Infrared (LUVOIR) mission study. On the right we see the individual segments of the primary. This telescope primary exhibits three diffraction gratings which diffract light from the much brighter star across the image plane and create background noise for imaging and spectroscopy of exoplanets. The direction of the "rulings" of the gratings are shown using red, blue and yellow arrows.

\section{The LUVOIR pupil has several-rings of Hex's}

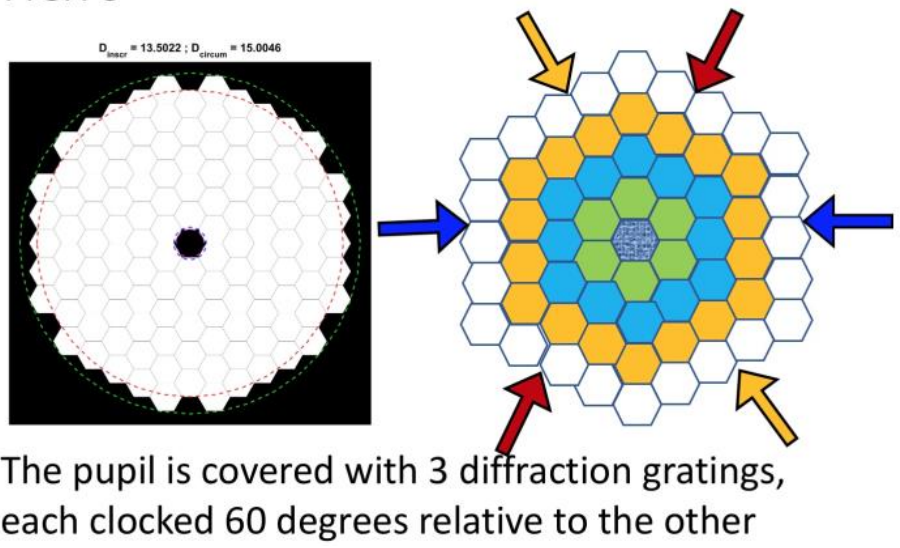

Figure 4 LUVOIR pupil left and its partitioning into three linear diffraction gratings. The 3 "ruling" directions are clocked 60-degrees in relation to each other. The directions of the grating rulings are shown by the arrows in the three colors: red, blue and yellow. 
The rulings are discontinuous across the LUVOIR pupil, but that does not make a difference to the diffractive properties of the straight lines. Figure 5 shows 2 of the 3 sets of diffraction grating rulings across the hexagonally segmented LUVOIR pupil. The third set of rulings, the horizontal set are not show to avoid confusion in the drawing.

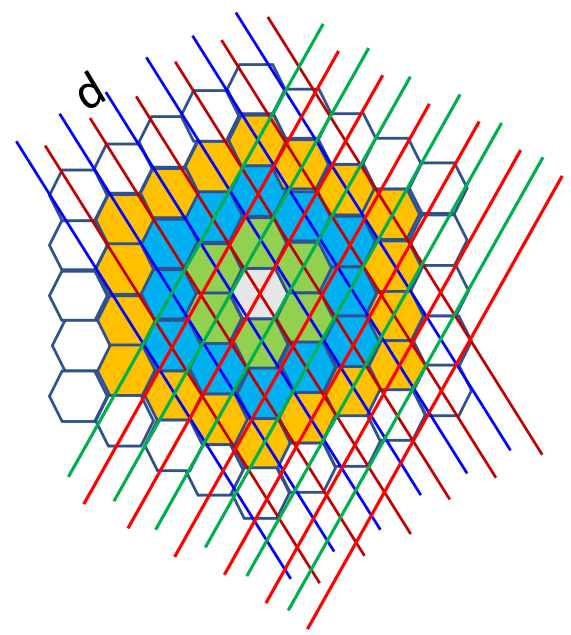

Figure 5 Two of the three diffraction gratings across the close-packed hexagon-segmented primary mirror are shown. The spacing of the "ruling" is $d$.

Figure 5 shows the close-packed hexagon-segmented primary mirror with lines drawn to show the grating "rulings" and the direction of the rulings. Note that to keep confusion down we have not drawn in the set of horizontal "rulings". The "groove-spacing" is seen to be $d$, where $d$ is one-half the face-to-face distance across the individual regular hexagons. The diffraction causes a structured background across the image plane that may obscure important exoplanets and may introduce unwanted polarization aberrations into the coronagraph to affect image quality.

\section{The PSF for a monochromatic star}

Gratings diffract light into orders which map a single on-axis point (a monochromatic star, for example) into multiple images of that monochromatic star. If the source is polychromatic then the grating maps the polychromatic single on-axis point into multiple spectral images stretched out radially.

Let $\lambda$ =wavelength; $\mathrm{d}=$ ruling spacing; $\mathrm{n}=$ diffraction order and $\theta$ =angle from the ax is, then the grating equation that relates these 4 variables is written:

$$
\begin{aligned}
& n \lambda=2 d \sin (\theta) \\
& \text { or for very small angles we can write } \\
& \theta=\frac{n \lambda}{2 d} \text {, where } \theta \text { is in radians, }
\end{aligned}
$$

Eq 1

The angular separation between diffraction orders is as given in Eq. 1 above.

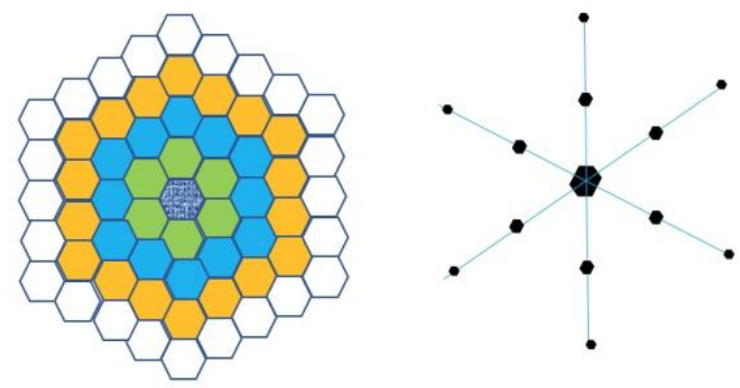

Figure 6 shows, left, a pupil map and right a representation of the monochromatic PSF associated with the close-packed hexagon-segmented primary mirror shown on the left. The points on the right-hand side show the location of the diffraction orders. The center is the image of the star and the first ring of points corresponds to the $1^{\text {st }}$ order of the three diffraction gratings. The second ring of points corresponds to the 2 nd order of the three diffraction gratings. 
In figure 6 we see that a Lyot coronagraph occulting mask would only block light from the zero-diffraction order, which contains light from the bright central star. But light from the higher diffraction orders $\mathrm{n}>1$ will scatter around the occulting mask to flood the detector plane. The occulting mask could be designed such that each order has its own mask, but that would block portions of the FOV where exoplanets might be found.

To determine if the diffraction images of the parent star will obscure exoplanets, we calculate the angular separation between zero order and the first order, $\mathrm{n}=1$, for polychromatic light. Table 2 below shows the angular separation of the 450, 500, $550 \mathrm{~nm}$ wavelength monochromatic diffraction orders as a function of the face-to-face segment size.

Table 2 Angular separation of the diffraction orders for face-to-face segment sizes: 1,2,3,4 meters. This applies to the diffraction orders shown in Fig. 6, above.

\begin{tabular}{|c|c|c|c|}
\hline $\begin{array}{c}\text { Face-to Face segment } \\
\text { size in meters }\end{array}$ & $\begin{array}{c}\text { Angle for 450-nm } \\
\text { masec }\end{array}$ & $\begin{array}{c}\text { Angle for 500-nm } \\
\text { masec }\end{array}$ & $\begin{array}{c}\text { Angle for 550-nm } \\
\text { masec }\end{array}$ \\
\hline 1 & 93 & 103 & 113 \\
\hline 2 & 47 & 52 & 57 \\
\hline 3 & 31 & 34 & 38 \\
\hline 4 & 23 & 26 & 28 \\
\hline
\end{tabular}

We compare the entries in Table 2 with the entries in Table 1 and see that the unwanted diffraction images of the parent star fall within the same FOV region as the exoplanets. Clearly there would be a significant advantage to the development of a straightforward, low absorption way to eliminate these diffraction orders. The pinwheel pupil provides that opportunity.

\section{The PSF for a polychromatic star}

Exoplanets are very faint thermal sources. If they are to be observed in monochromatic or narrow band light integration times become impossibly long. The HabEx coronagraph is planned to observe in $100 \mathrm{~nm}$ bandwidths. One of these bandwidths in 450 to $550 \mathrm{~nm}$ and we have used those values to compute the diffraction angles shown in Table 2. The star image at $\mathrm{n}=1$ for the 1 -meter face-to-face segments is a colored radial streak or small spectrum with $450 \mathrm{~nm}$ light at $93 \mathrm{masec}$ and $550 \mathrm{~nm}$ light at 113 masec. The 2-meter face-to-face segments is a colored radial streak or small spectrum with $450 \mathrm{~nm}$ light at 47 masec and $550 \mathrm{~nm}$ light at 57 masec. This continues to the 4-meter face-to-face segments which give a colored radial streak or small spectrum with $450 \mathrm{~nm}$ light at 23 masec and $550 \mathrm{~nm}$ light at 28 masec.

\section{Isoplanatic point spread function for image processing}

The polychromatic PSF shown in figure 6 (right) not linear shift invariant and is therefore the optical system is not isoplanatic. Also, looking at Fig 6, right, we see that the PSF is not rotationally symmetric either. These two facts complicate digital image processing. In this paper, we have devised a pupil segmentation or topology architecture that will produce images from an emulated filled aperture telescope pupil even though the pupil is mechanically segmented. This promises to reduce significantly the effects of an anisoplanatic PSF and will make digital image processing more reliable and less uncertain.

\section{Compensating for hexagonal segments}

Technologies to compensate for the diffraction patterns produced by straight line gaps and straight-line support structures across primary mirrors of large telescopes has been an area of active study recently ${ }^{8},{ }^{9},{ }^{10},{ }^{11},{ }^{12},{ }^{13}$. None of these methods may be completely satisfactory, however, since light is absorbed in the process.

\section{CURVED SECONDARY SUPPORT STRUCTURES NOT NEW}

\section{Background}

Breckinridge (2018) ${ }^{14}$ suggested partitioning the primary into curved sided segments and curving the secondary support structures to reduce diffraction noise at its source to control diffraction noise at the image plane of exoplanet coronagraphs. We have shown above that the hexagonal segment architecture or pupil topology leads to unwanted diffraction noise in the system. It is good engineering practice to seek ways to eliminate or reduce "noise" at its source, rather than devise complicated and signal absorbing methods to compensate. Methods to mitigate diffraction noise were developed over the years by amateur astronomers, and later optical scientists. However, the professional space and ground astronomical optical telescope and instrument community seems not aware of these techniques. 
C. H. Werenskiold $(1941)^{15}$ reported on the work of A. Couder published in the French journal: Astronomy, Jan 1934 and translated into English and republished in Amateur Telescope Making Advanced (scientific American Publishing), pp 620-622. Couder proposed controlling diffraction in Newtonian and Cassegrain type telescopes by placing lune shaped curved masks over the straight edge support structure of the secondary. These masks blocked significantly, the light-gathering ability of the telescope, negatively affecting the telescope transmittance. Werenskiold proposed curving the secondary support structures themselves as shown below in Fig 7 to reduce masking of the primary mirror and control the diffraction spikes.
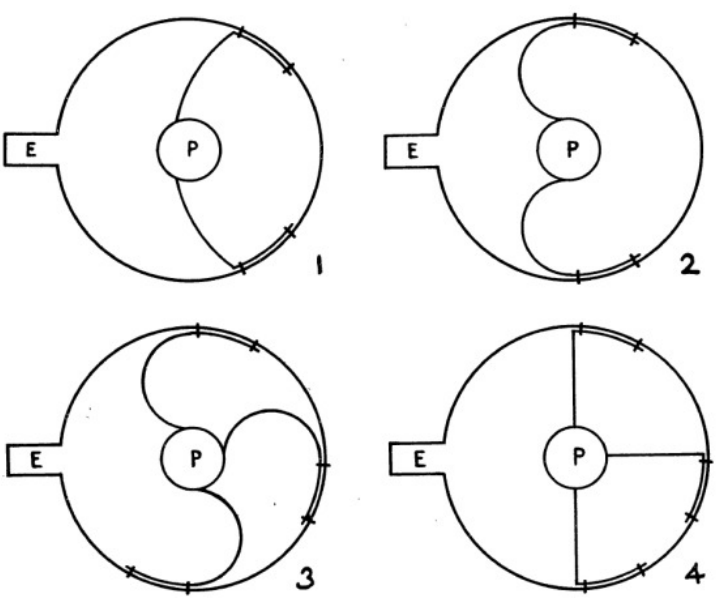

Figure 7 The four secondary support structures built by Werenskiold ${ }^{14}$ and used for visual observation of planets. View looking from the open end of a reflecting telescope back toward the primary mirror. He reports that \#4 gives the lowest quality image and that curving the support structure appears to remove the diffraction spikes from visual images to give higher contrast for visual planetary observations.

Werenskiold writes:

"It is generally conceded that a reflector, in regard to definition obtained, is apt to be somewhat inferior to a refractor of comparable size. However, the use of a curved spider in a reflector appears to be a promising step towards reducing this difference in comparative performance of the two telescope types."

\section{MODELLING A 10-METER PINWHEEL PUPIL}

\section{Pupil architecture of topology}

Richter ${ }^{16}$ selected 6 diffraction masks and photographed the diffraction pattern from each to show that curved arcs on the pupil left no discernible diffraction pattern at the image plane. Harvey ${ }^{17}$ (in this volume) used FRED $^{18}$ and applied the design methodology outlined in Richter ${ }^{15}$ and developed further by Harvey and Ftaclas ${ }^{19}$ along with the computer analysis program FRED to show that the image plane diffraction patterns from curved secondary support structure is less than $10^{-6}$ where-as the image plane diffraction patterns from straight line secondary support structure are $\sim 10^{-2}$.

Based on our intuitive understanding of diffraction from curved segments we designed a pupil topology for a "first look" at the diffraction effects. The design we chose is shown in Figure 8 below. We selected a 10-meter Cassegrain primary with an obscuration ratio of 0.16 and six curved secondary mirror support struts, each with a $30^{\circ}$ arc of a circle and $20 \mathrm{~mm}$ wide gaps. There are three rings or zones of segments curved on all sides. Each zone contains 12 curved sided segments to create a telescope entrance pupil that has 36 segments. 


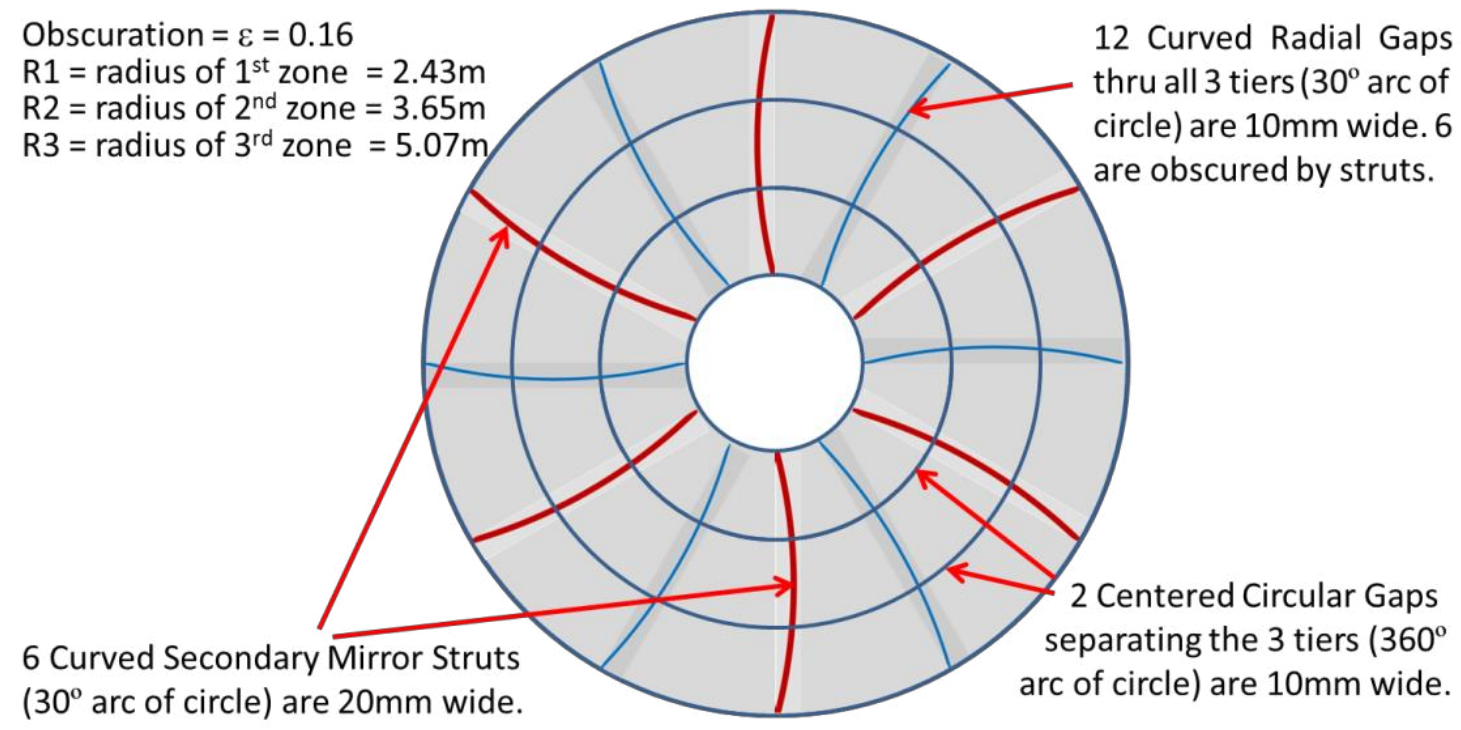

Figure 8 Cassegrain primary with an obscuration ratio of 0.16 and six curved secondary mirror support struts (shown in Red), each with a $30^{\circ}$ arc of a circle and and $20 \mathrm{~mm}$ wide. There are three rings or zones of segments curved on all sides. Each zone contains 12 curved sided segments to create a telescope entrance pupil that has 36 segments.

$$
f(\phi) \text {, for } \theta=0.75 \text { arc-sec. }
$$

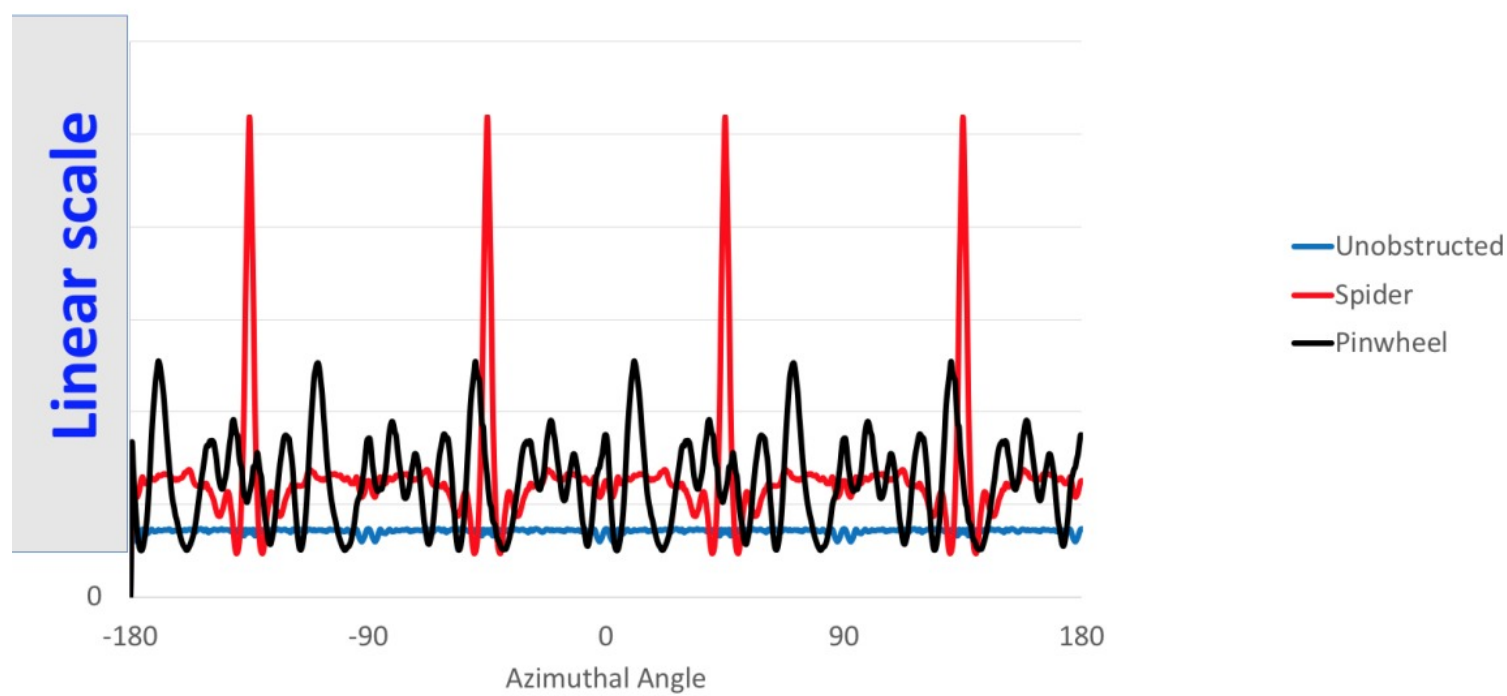

Figure 9 Image plane linear-scale monochromatic intensity distribution from three 10-m diameter pupil topologies: unobstructed, spider and pinwheel are shown at field radius 0.75 arc-second for azimuth angles -180 to +180 degrees.

Figure 9 shows a plot of monochromatic intensity, on a linear scale, as a function of azimuth angle at field 0.75 arc sec. for three 10-m diameter pupil architectures: unobstructed, spider and pinwheel. The computation was performed using MatLab. Computational capacity limited the size of the sample interval across the pupil and we believe that may have resulted in an incorrect representation of the image plane diffracted light. However, several features in Fig 9 are worth noting. The profile for the 4-spider mask, shown in red, is much higher than that for the pinwheel which 
indicates that the pinwheel pupil is making a contribution to smoothing out the diffraction pattern. The prominent dip in energy at the feet of the spider diffraction pattern is probably the result of the very narrow bandwidth of this monochromatic computation. The noise on the pinwheel is probably caused by having an insufficient number of samples across the pupil, which was dictated by the array sizes and the computational time limits of Matlab.

Consequently, we decided to drop MatLab as our computational tool and turn to Photon Engineering. LLC and the FRED software. Computations using FRED were successful and are shown in the paper: SPIE Proc 10698-60 ${ }^{17}$.

\section{MANUFACTURE OF CURVED-SIDED SEGMENTS}

The manufacture of curved-sided non-circular aspheric-surface segments is not different than the manufacture of hexagonal-sided aspheric-surface segments, provided the radius of curvature of the sides are gentle, like those shown in Fig 8. The biggest challenge is maintaining the "global" optical surface figure for those regions near the "points" of each segment. Technology developed by Tinsley for the figuring of the Keck hexagonal-sided segments included: stressed mirror polishing and deterministic polishing is applicable to the curved sided segments. One approach is given here.

With a full-sized tool use rapid material removal polish for the roundels, removing most of the volume between the "nearest sphere" and the off-axis aspheric form. Then shape the roundel into a curved sided segment and remove the remaining small volume of surface error with deterministic small tools. The curved-sided segment is then finely polished with deterministic tools as was done for the 18 hexagonal segments of the JWST primary mirror.

Pinwheel mirror segments may be made of a number of different materials. For example, SCHOTT offers extremely stable monolithic mirror substrates of ZERODUR. These have been aggressively light-weighted up to 4$\mathrm{m}$ in diameter. A pinwheel segment can be undercut around its perimeter, as shown in Figure 10 below. The steps to processing would be: 1 . cut the roundel, 2. mill to near optical shape, 3. lightweight by removing up to $90 \%$ material, leaving "lands" for mounting, and 4 , acid etch to mitigate subsurface damage. The segment would be lightweighted as an isogrid (not the rectangular grid pattern shown in Figure 10) to minimize mass and give maximum strength for launch and alignment stability. After optical fabrication as an off-axis roundel the petal would be parted out by machining.

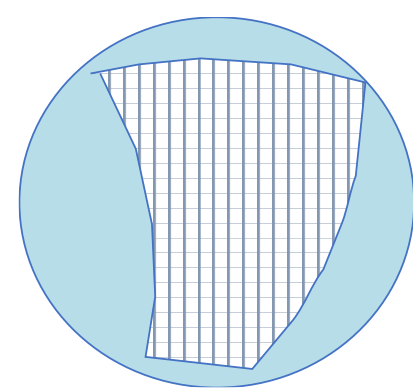

Figure 10 Pinwheel pupil segment is shown within its Zerodur roundel. The rectangular grid support structure is shown to indicate an isogrid back structure. Engineering details of this isogrid structure would follow detailed structural engineering for thermal, mechanical, and structural design of the mirror as a space-flight element attached to a back-plane.

\section{ACKNOWLEDGEMENT}

We wish to acknowledge the work on the Pinwheel Pupil, performed by Dr. Kathryn Jackson during her Post Doc assignment at Caltech. This work was supported in part by a NASA TDEM15 research grant \#NNX17AB29G awarded by NASA Science Mission Directorate to the College of Optical Sciences at the University of Arizona. This work was also supported in part by a NASA contract \# NNX17AD08G awarded by STMD, Early Stage Innovation program to Professor Pellegrino of Caltech.

\section{REFERENCES}

\footnotetext{
${ }^{1}$ Polidan, R., J. B. Breckinridge, C, F, Lillie, H. A. MacEwen, M. R. Flannery and D. R. Dailey (2016) "Innovative telescope architectures for future large space observatories", J. Astron. Telesc. Instrum. Vol 2

${ }^{2}$ Harvey, J. E. ; R. G. Irvin, K. Crabtree, R. N. Pfisterer and J. B. Breckinridge (2018); "Diffraction Analysis of Large Segmented Mirror Concepts for Exoplanet Exploration" SPIE Proc. 10698-60
} 
3 Trunbull, M. C., ExoCat-1: The Nearby Stellar Systems Catalog for Exoplanet Imaging Missions, $\underline{\operatorname{arXiv}: 1510.01731}$ [astroph.SR] arXiv:1510.01731 [astro-ph.SR] 2015.

${ }^{4}$ Beckwith, S. V. W.; M. Stailelli, A. M. Koekemoer, J. A. R. Calswell, et. al.; "The Hubble Ultra Deep Field” Astron. J. 132:1729-1755 (2006).

${ }^{5}$ Breckinridge, J. B., T. G. Kuper and R. V. Shack "Low-scattered light camera: a model” Optical Engineering 23, pp 816-820; (1984)

${ }^{6}$ Harvey, J. E., R. N. Pfister and J. B. Breckinridge, "The role of narrow angle forward surface scatter and particulate scatter in exoplanet exploration", Proc SPIE 10698-188, 2018.

${ }^{7}$ Troy, M. and G. Chanan, "Diffraction effects from giant segmented-mirror telescopes," Appl.Opt.42,3745-3753(2003). 30meter diameter CELT telescope, (renamed the Thirty Meter Telescope or TMT which uses 1.44 meter segments)

${ }^{8}$ Pueyo, L., and Norman, C., High Contrast Imaging with an arbitrary aperture: active compensation of aperture discontinuities. The Astroph. Journ., 769:102 (31pp), doi:10.1088/0004-637X/769/2/102 C_2013.

${ }^{9}$ Garreth Ruane, Dimitri Mawet, Jeffrey Jewell, Stuart Shaklan, "Performance and sensitivity of vortex coronagraphs on segmented space telescopes," Proc. SPIE 10400, 104000J ; doi: 10.1117/12.2274508 (2017)

10 Jewell, J. G. Ruane, S. Shaklan, D. Mawet, D. Redding, "Optimization of coronagraph design for segmented aperture telescopes," Proc. SPIE 10400, 104000H; doi: 10.1117/12.2274574 (2017)

11 Vanderbei, R.J., Spergel, D.N., and Kasdin, J.N., "Spiderweb Masks for High Contrast Imaging,: ApJ vol. 590, 593, 2003.

${ }^{12}$ Lillie, C., M. Flannery, D. Dailey “Segmented Telescopes For Coronagraphs” Proc of SPIE Vol. 5487 0277-786X/04/\$15 . doi: $10.1117 / 12.552348(2004)$

${ }^{13}$ Alexis Carlottia,b, Dimitri Mawetc, Laurent Pueyo Optimal apodizers for the vector vortex coronagraph with on-axis telescopes, Proc. of SPIE Vol. 8864, 88641P; CCC code: 0277-786X/13/\$18 · doi: 10.1117/12.2024511

${ }^{14}$ Breckinridge, J. B. "The pinwheel pupil discovery: exoplanet science and improved processing with segmented telescopes" Paper \# 439.04 231st meeting of the American Astronomical Society, Washington, DC. 2018.

${ }^{15}$ C. H. Werenskiold (1941) "Improved Telescope Spider Design”, Journ. Royal Astron. Soc. Canada, 35, 268-272. AND C. H. Werenskiold, “A note on curved spiders,'” Sky Telesc. 262-263, 1969

${ }^{16}$ Richter, J. L. (1984) "Spider diffraction: a comparison of curved and straight legs", AO, 23 1907-1913

${ }^{17}$ Harvey, J. E. , R. G. Irvin, K. Crabtree, R. N. Pfister and J. B. Breckinridge "Diffraction Analysis of Large Segmented Mirror Concepts for Exoplanet Exploration", SPIE proceedings 10698-060, 2018.

${ }^{18}$ J. E. Harvey, R. G. Irvin and R. N. Pfisterer, "Modeling Physical Optics Phenomena by Complex Ray-tracing", Opt. Eng., 54 (3), 035105 (2015). doi: 10.1117/1.OE.54.3.035105.

${ }^{19}$ Harvey, J. E. and C. Ftaclas, "Diffraction Effects of Telescope Secondary Mirror Spiders upon Various Image Quality Criteria”, Appl. Opt. 34, 6337-6349 (1995). 